\title{
Accuracy of breast MRI in patients receiving neoadjuvant endocrine therapy: comprehensive imaging analysis and correlation with clinical and pathological assessments
}

\author{
Joana Reis ${ }^{1,2}$ - Jonas Christoffer Lindstrøm ${ }^{2,3} \cdot$ Joao Boavida $^{1} \cdot$ Kjell-Inge Gjesdal $^{1,4} \cdot$ Daehoon Park $^{5}$. \\ Nazli Bahrami ${ }^{6,7,8}$. Manouchehr Seyedzadeh ${ }^{1} \cdot$ Woldegabriel A. Melles $^{1} \cdot$ Torill Sauer $^{2,8,9} \cdot$ Jürgen Geisler ${ }^{2,7,8}$. \\ Jonn Terje Geitung ${ }^{1,2}$
}

Received: 27 May 2020 / Accepted: 31 July 2020 / Published online: 12 August 2020

(c) The Author(s) 2020

\begin{abstract}
Purpose To assess the accuracy of magnetic resonance imaging (MRI) measurements in locally advanced oestrogen receptor-positive and human epidermal growth factor receptor 2-negative breast tumours before, during and after neoadjuvant endocrine treatment (NET) for evaluation of tumour response in comparison with clinical and pathological assessments. Methods This prospective study enrolled postmenopausal patients treated neoadjuvant with letrozole and exemestane given sequentially in an intra-patient cross-over regimen. Fifty-four patients were initially recruited, but only 35 fulfilled the inclusion criteria and confirmed to participate with a median age of 77 . Tumours were scanned with MRI prior to treatment, during the eighth week of treatment and prior to surgery. Additionally, changes in longest diameter on clinical examination (CE) and tumour size at pathology were determined. Pre- and post-operative measurements of tumour size were compared in order to evaluate tumour response.

Results The correlation between post-treatment MRI size and pathology was moderate and higher with a correlation coefficient $(r) 0.64$ compared to the correlation between CE and pathology $r=0.25$. Post-treatment MRI and clinical results had a negligible bias towards underestimation of lesion size. Tumour size on MRI and CE had $0.82 \mathrm{~cm}$ and $0.52 \mathrm{~cm}$ lower mean size than tumour size measured by pathology, respectively.

Conclusions The higher correlation between measurements of residual disease obtained on MRI and those obtained with pathology validates the accuracy of imaging assessment during NET. MRI was found to be more accurate for estimating complete responses than clinical assessments and warrants further investigation in larger cohorts to validate this finding.
\end{abstract}

Keywords Breast cancer $\cdot$ Neoadjuvant therapy $\cdot$ Magnetic resonance $\cdot$ Tumour response

\section{Abbreviations}

3D

AIs

AJCC

BCS

BI-RADS ${ }^{\circledR} \quad$ Breast imaging-reporting and data system

Jürgen Geisler and Jonn Terje Geitung are equally contributed.

Electronic supplementary material The online version of this article (https://doi.org/10.1007/s10549-020-05852-7) contains supplementary material, which is available to authorized users.

Joana Reis

joana.reis@ahus.no; joanaroquedosreis@gmail.com

Extended author information available on the last page of the article

$\begin{array}{ll}\text { CE } & \begin{array}{l}\text { Clinical examination } \\ \text { CI }\end{array} \\ \text { DCIS } & \begin{array}{l}\text { Donfidence interval } \\ \text { Ductal carcinoma in situ } \\ \text { DWIffusion-weighted imaging, single-shot, } \\ \text { echo planar imaging }\end{array} \\ \text { EPI } & \begin{array}{l}\text { Echo planar imaging } \\ \text { Eestrogen receptor }\end{array} \\ \text { HER-2 } & \text { Human epidermal growth factor receptor } \\ \text { LABC } & 2 \\ \text { LD } & \text { Locally advanced breast cancer } \\ \text { MRI } & \text { Longest diameter } \\ \text { NAAI } & \text { Magnetic resonance imaging } \\ \text { NET } & \text { Neoadjuvant aromatase inhibitor } \\ \text { O.d. } & \text { Neoadjuvant endocrine treatment } \\ \text { PR } & \text { Once a day } \\ \end{array}$


r Correlation coefficient

RECIST Response evaluation criteria in solid tumours

TFE Turbo field echo

TNM Tumour, node and metastasis

TSE Turbo spin echo

UICC Union for International Cancer Control

\section{Introduction}

Current response assessments of locally advanced breast cancer (LABC) in the neoadjuvant setting are not entirely accurate in determining pathological complete response, depending on the modality used, the measurement technique and varied response of different tumour subtypes [1, 2]. Tailoring the most accurate tumour size after neoadjuvant treatment must require a precise assessment, in order to achieve the most appropriate surgical management: mastectomy or breast-conserving surgery (BCS). Conventional assessment includes clinical examination (CE) and imaging modalities (mammography, ultrasound and/or magnetic resonance imaging (MRI)), along with pathological examinations of the sectioned surgical specimen, which take place typically during the first weeks after surgery.

Performed by a trained physician, CE is a widely recognized assessment for detecting breast cancers and for tumour size monitoring [3]. CE is a non-invasive, low-cost method, without the use of ionizing radiation $[2,4]$. For patients with breast symptoms, a CE should be performed before any additional imaging assessments are sought. Additionally, it should be a part of routine periodic examinations, especially in older women (more than 69 years old) and in women less than 50 years who are less likely to undergo mammographic screening [3]. CE is typically performed prior to each neoadjuvant therapy cycle or every 4-6 weeks during neoadjuvant endocrine therapy (NET).

Although mammography and ultrasound are reliable methods to analyse tumour size at diagnosis, changes within the tumour after neoadjuvant therapy may be difficult to assess. Targeted ultrasound is mandatory for malignant breast tumours and of the axilla for clinical staging if neoadjuvant therapy is planned [2]. Ultrasound has been revealed to be a better predictor for pathologic tumour size than mammography after treatment with neoadjuvant therapy. However, ultrasound is operator dependent, and its accuracy varies $[2,5,6]$. Mammography has been less specific than $\mathrm{CE}$ for detecting presence of residual tumour after therapy, and it adds challenges with mammographically occult tumours and microcalcifications which do not correlate with presence of viable tumours [2]. Furthermore, mammography has the disadvantage of being less accurate in high density breasts.
Dynamic contrast-enhanced MRI is the most sensitive of available imaging modalities in characterization of breast malignant tumours as a functional technique that allows the evaluation of residual viable tumour after neoadjuvant therapy by detecting changes in tumour vascularity [7-14]. Multiple studies have clarified that breast MRI is the most accurate imaging modality to determine disease response to neoadjuvant chemotherapy, with sensitivity approaching $90 \%$ and specificity of $60 \%$ to $100 \%$, and it is particularly helpful in patients with confirmed multifocal and multicentric tumours on the pre-treatment study [1, 2, 4, 7, 8, 15-17]. Breast MRI has been considered to have a role in guiding breast cancer surgery by differentiating residual tumour from pathological complete response after neoadjuvant therapy, and planning resections to achieve clear margins in BCS $[11,18]$.

Response Evaluation Criteria in Solid Tumours (RECIST) guidelines recommend assessment of the largest tumour diameter [19]. Overestimation of tumour size may lead to exceedingly radical surgery and poorer cosmetic and psychosocial outcomes; underestimation may lead to involved surgical margins, and apparent radical surgery, though with missed additional tumour foci, eventually leading to a reoperation [20].

No previous research has investigated the performance of clinical and MRI assessment methods in the evaluation of residual tumour size in patients receiving combined neoadjuvant endocrine therapy with third generation aromatase inhibitors [21, 22]. Aromatase inhibitors (AIs) are well-established and low-toxicity drugs to improve surgical outcomes in LABC in elderly or otherwise fragile and postmenopausal patients, particularly in selected patients with oestrogen receptor (ER) highly $(>50 \%)$ positive disease [23-28]. Compared to neoadjuvant chemotherapy, AIs have demonstrated comparable outcomes in postmenopausal patients with ER positive LABC [29-32]. Likewise, several clinical randomized trials have confirmed that third generations AIs (letrozole, anastrozole, exemestane) are preferred over ER antagonists such as tamoxifen, due to higher clinical and radiological response rates [33-35]. Nonetheless, tumours respond differently to neoadjuvant AIs and not all patients respond in a satisfactory way, sometimes even warranting additional neoadjuvant chemotherapy.

The current lack of published studies to assess the accuracy of MRI in predicting residual size of breast tumours following NET opens opportunities for evidence-based studies [10]. There have been many published studies determining the diagnostic advantages of MRI versus clinical current assessments to evaluate the tumour response after neoadjuvant chemotherapy. Although, it is important to understand the different mechanisms of action between chemotherapy and endocrine therapy, and to recognize that tumours profiles and tumours stages are distinct. As neoadjuvant therapy, 
especially endocrine therapy, for breast cancer continues to move beyond locally advanced disease, efforts such as this one will be imperative to guide daily clinical practice, embracing both the available high-level evidence and guidelines to investigate current assessments for evaluating tumour response to neoadjuvant therapy.

The aim of the current study is to compare tumour size estimated by CE and MRI with the tumour size in surgical specimen, in order to determine which is the most accurate assessment to evaluate tumour response in patients with LABC treated neoadjuvant with aromatase inhibitor (NAAI) therapy.

\section{Materials and methods}

\section{study population}

From February 2015 to November 2019, patients with biopsy-proven locally advanced oestrogen receptor (ER)positive breast cancer and human epidermal growth factor receptor 2 (HER-2)-negative status suitable for NET were eligible for this prospective, open-label, randomized sub-study of the Neoletexe trial, (Fig. 1) [36]. All participants had to be postmenopausal to benefit from aromatase inhibition. Figure 2 reports a flowchart of the study cohort in which 54 patients were initially recruited, but only 35 fulfilled the inclusion criteria and confirmed to participate. Thirty-four of the patients studied were female and only one male with a total median age at diagnosis of 77 years, and a mean age of 74.3 years.

The exclusion criteria were triple-negative breast cancer, life-threatening metastasis, previous therapy for breast cancer within the last 12 months and/or medications that may interfere with endocrine therapy, inconclusive imaging,

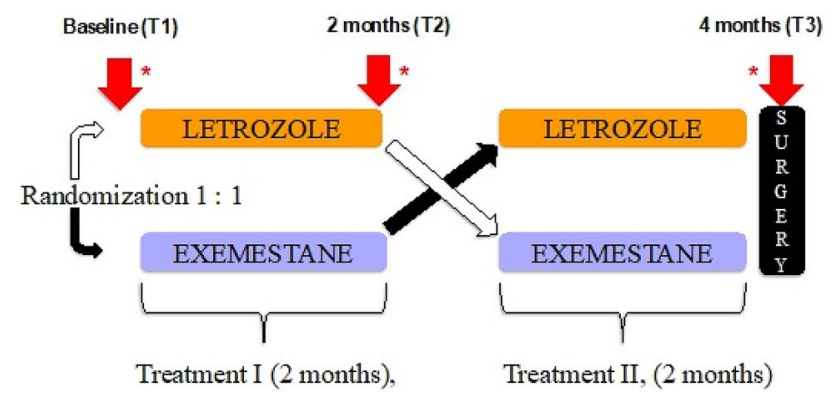

Fig. 1 Design of the Neoletexe trial: a prospective, randomized intra-patient cross-over study. *Time points for breast magnetic resonance imaging examinations, open surgical biopsies of breast tumour (acquired after MRI examinations at baseline and after 2 months of treatment; and the third biopsy after 4 months of treatment and after surgery) and blood samples oncological and/or pathological measurements (Supplementary Table 1).

The diagnosis of breast cancer was established after a digital mammography, accompanied by breast and axillary ultrasound, a stereotactic or ultrasound- guided breast biopsy and CE. Primary tumours were classified using clinical and radiological tumour, node and metastasis (TNM) staging system for breast cancer, according to the classification system proposed by the American Joint Committee on Cancer (AJCC, 2017) and Union for International Cancer Control (UICC, 2017). All patients, as part of their clinical care, were screened for distant metastasis with thoracic, abdominal and pelvic computed tomography scan and bone scintigraphy. Only two patients presented with limited systemic metastasis at baseline were allowed to enrol. Table 1 includes the characteristics of included patients $(n=35)$.

\section{Imaging}

MRI scans were performed prior to the start of NAAI therapy (baseline), 8 weeks after the first cycle and prior to the second cycle of therapy (second examination), after the final administration of NAAI therapy and immediately before surgery (third examination) (Fig. 1). Imaging was acquired using a Philips Ingenia 1.5 T scan (Philips Healthcare, Best, Netherlands). A dedicated sixteen- channel bilateral breast coil with parallel imaging capabilities was applied. The MRI protocol consisted prior to the administration of contrast of an axial turbo spin echo (TSE) T1-weighted sequence, an axial single-shot echo planar (SS-EPI) diffusion-weighted imaging with two respective $b$ factors $(50,800)$ (DWI SShEPI) and a axial 3-dimensional (3D) T2-weighted with fat suppression. Two dynamic sequences where then applied in an interleaved pattern prior (for baseline acquisition data) and during the injection of the contrast agent. The high temporal resolution images were acquired using a 3D TI_T2* weighted multi-echo planar imaging (EPI) sequence and intercalated with a dynamic high spatial resolution 3D T1-weighted turbo field echo (TFE) sequence. Both contrastenhanced imaging sequences include a total scanning time of 7 min with a full coverage of both breasts with no slice gap. More details on the breast MRI sequences are provided in Table 2. Diagnostic mammography and ultrasound examinations were performed at baseline according to the routine practice of our institution; mammographic and sonographic results were therefore not included in the comparison analysis. The institutions breast imaging radiologists interpreted these examinations, according to the American College of Radiology Breast Imaging- Reporting and Data System (BI-RADS®) lexicon (ACR BI-RADS® Atlas 2013, https ://www.acr.org/Clinical-Resources/Reporting-and-DataSystems/Bi-Rads). 
Fig. 2 Flowchart of participants through the study including the patient's enrolment and exclusion criteria. *Non-adherence to MRI examinations: refusal MRI or refusal to complete MRI examination (e.g. claustrophobia, and/or anxiety); imaging quality does not meet the diagnostic requirements: poor image quality (e.g. motion artefacts, chemical shift artefacts, and inadequate image contrast). $L D$ Longest diameter, $M R I$ magnetic resonance imaging, $n$ number of patients

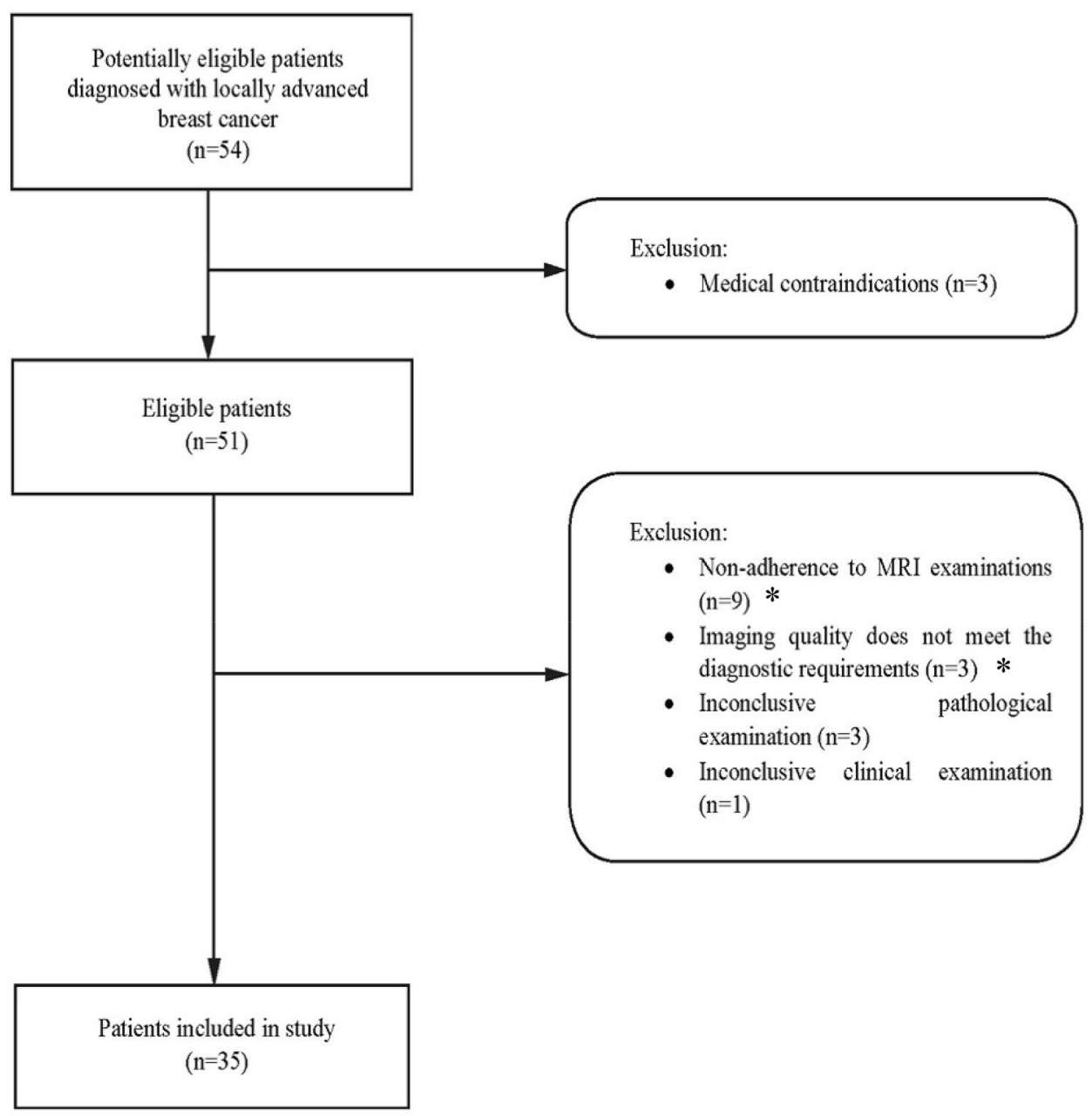

\section{Clinical assessment}

Clinical tumour assessments were conducted at the oncological outpatient clinic by the same medical breast cancer oncologist every 4 weeks during the entire study, but only evaluations performed at baseline, following 8 weeks and 16 weeks were included in this analysis. The NAAI intra-patient cross-over regimen consisted of one of the following treatment arms: letrozole $2.5 \mathrm{mg}$ o.d. for at least 8 weeks thereafter continuing with exemestane $25 \mathrm{mg}$ o.d. for another 8 weeks prior to surgery; and exemestane $25 \mathrm{mg}$ o.d. for at least 8 weeks thereafter continuing with letrozole $2.5 \mathrm{mg}$ o.d. for 8 weeks prior to surgery (Fig. 1) [36]. Calliper measurements of maximum tumour size (in centimetres) and tumour quadrant location were recorded. Afterwards, response categories were assessed and categorized into four groups: complete response, partial response, progressive and stable disease. Complete clinical response was defined as no palpable lesions. Partial clinical response was defined as a decrease in the longest diameter (LD) of primary tumour of at least a $30 \%$. Progressive disease was defined as at least a
$20 \%$ increase in the LD of the primary tumour. Stable disease was defined as neither partial response nor progressive disease. Collection of blood samples was performed before each time point (Fig. 1). Upon completion of NAAI therapy, all patients underwent surgery following $\mathrm{CE}$ by an experienced breast surgeon, and treated with adjuvant therapy using letrozole $2.5 \mathrm{mg}$ o.d. for a period of at least 5 years as standard recommendation. Patients with progressive or stable response during or after NAAI therapy were offered additional treatment options according to the national guidelines without any restriction.

\section{Image analysis}

Image evaluation included both qualitative, consistent with the standard BI-RADS $®$ and TNM classifications, and quantitative imaging interpretations. All images were independently analysed by two breast imaging radiologists (one with more than 10 years and the other with 2 years of experience in interpreting breast MRI), and reviewed afterwards in a consensus interpretation. The radiologists were 
Table 1 Characteristics of the analysis group $(n=35)$

\begin{tabular}{|c|c|}
\hline \multirow[t]{2}{*}{ Characteristics } & Analysis group $(n=35)$ \\
\hline & Postoperative \\
\hline
\end{tabular}

Age

Median 77

Gender

Female $\quad 34$

Male 1

Menopausal/andropause status

Postmenopausal 34

Andropause 1

Clinical exam

Palpable

Skin retraction

Yes

No

Nipple retraction

Yes

No

Skin thickening

Yes

No

Clinical tumour stage

$\mathrm{T} 2$

T3

T4

Clinical nodal status

N0

N1

Imaging (MRI) tumour stage $\mathrm{T} 2$

T3

$\mathrm{T} 4$

Imaging (MRI) nodal status

NO

$\mathrm{N} 1$

N2

Oestrogen receptor status

Positive

Negative

Analysis not performed

Progesterone receptor status

Positive

Negative

Analysis not performed

HER-2 status

Negative

Positive

Analysis not performed

Invasive histologic findings

Ductal carcinoma, NST

34

35

NA

NA

20

15

NA

12

23

23

12

6

29

28

7

NA

1

4

30

NA

25

9

1

$35 \quad 3$

0

0

NA

NA

NA
1 (continued)

\begin{tabular}{|c|c|c|}
\hline \multirow[t]{2}{*}{ Characteristics } & \multicolumn{2}{|c|}{ Analysis group $(n=35)$} \\
\hline & Baseline & Postoperative \\
\hline Lobular carcinoma & 5 & 8 \\
\hline Papillary & 1 & 0 \\
\hline Mucinous & 1 & 1 \\
\hline $\mathrm{pCR}$ & NA & 3 \\
\hline \multicolumn{3}{|l|}{ DCIS present $(*)$} \\
\hline No & 32 & 21 \\
\hline Yes & 3 & 14 \\
\hline \multicolumn{3}{|l|}{ LCIS present $(*)$} \\
\hline No & 34 & 30 \\
\hline Yes & 1 & 5 \\
\hline Surgery type & NA & \\
\hline Breast- conserving surgery & & 2 \\
\hline Mastectomy & & 33 \\
\hline Pathologic tumour stage & NA & \\
\hline T0 & & 2 \\
\hline $\mathrm{T} 1$ & & 8 \\
\hline $\mathrm{T} 2$ & & 22 \\
\hline $\mathrm{T} 3$ & & 2 \\
\hline Tis & & 1 \\
\hline Pathologic nodal status & NA & \\
\hline No & & 15 \\
\hline N1 & & 15 \\
\hline $\mathrm{N} 2$ & & 5 \\
\hline
\end{tabular}

CT Computed tomography, DCIS ductal carcinoma in situ, HER2 human epidermal growth factor receptor $2, n$ number of patients, $N A$ non-applicable, NST non-specific type, LCIS lobular carcinoma in situ, $p C R$ pathologic complete response

*In situ component at baseline was obtained based on diagnostic biopsy samples and at the post-operative time point based on full specimens

blinded to findings from clinical assessment and were not aware of the post-operative histopathology results. Table 3 shows the qualitative MRI findings at baseline. LD was measured as the greatest extent of disease. Response categories based on RECIST were assessed and categorized into four groups: complete response (no enhancement of all malignant lesions), partial response (decrease in the LD of primary tumour of at least a 30\%), progressive disease (at least a $20 \%$ increase in the LD of the primary tumour) and stable disease (no change). Contrast- enhanced images were analysed using Philips IntelliSpace Portal program (Philips Healthcare, The Netherlands). Manual assessment of tumour LD was performed through the tumour tracking software incorporated in Philips IntelliSpace Portal program at the point at maximum tumour enhancement (Fig. 3). 
Table 2 Technical parameters utilized for breast magnetic resonance sequences acquisition (Philips)

\begin{tabular}{|c|c|c|c|c|c|c|c|}
\hline Sequence & TR/TE (ms) & Flip angle & Dyn Reps & NSA & FOV $(\mathrm{mm})$ & $\begin{array}{l}\text { Slice thick- } \\
\text { ness }(\mathrm{mm})\end{array}$ & Acquisition time \\
\hline \multicolumn{8}{|l|}{ Pre- contrast } \\
\hline T1W-TSE & $487 / 8$ & NA & 1 & 2 & $280 \times 340$ & 3 & $2.40 \mathrm{~min}$ \\
\hline DWI SSh SE- EPI & $7000 / 103$ & NA & 1 & 3 & $280 \times 340$ & 3 & $5.50 \mathrm{~min}$ \\
\hline FS 3DT2W & $1300 / 145$ & NA & 1 & 1 & $370 \times 370$ & 2.2 & $5.20 \mathrm{~min}$ \\
\hline \multicolumn{8}{|l|}{ After a single injection of CA } \\
\hline 3DT1_T2* multi-echo EPI & $38 / 6.2 / 8.8$ & $28^{\circ}$ & 41 & 1 & $280 \times 340$ & 8 & $2,84 \mathrm{~s} /$ volume \\
\hline 3D T1W THRIVE & $5.4 / 2.6$ & $12^{\circ}$ & 6 & 1 & $360 \times 120$ & 2 & $56,5 \mathrm{~s} /$ volume \\
\hline
\end{tabular}

$C A$ Contrast agent, DWI SSh SE- EPI diffusion-weighted imaging, single- shot, echo planar imaging with two respective b factors (50, 800), $T 1 W$ THRIVE high spatial resolution 3D T1-weighted turbo field echo (TFE) sequence, EPI echo planar imaging, FOV field of view, NA non-applicable, NSA number of signals, $F S$ fat suppression, $T E$ time echo, $T R$ repetition time, TSE turbo spin echo, $T 1 W$ T1- weighted, $T 2 W$ T2- weighted, 3D 3-dimensional

Table 3 Few MRI findings based on ACR BI-RADS® Atlas Fifth Edition at baseline. Diverse associated features could be present at the same MRI

\begin{tabular}{|c|c|}
\hline MRI findings & $\begin{array}{l}\text { Analysis } \\
\text { group } \\
(n=35)\end{array}$ \\
\hline Mass & 27 \\
\hline \multicolumn{2}{|l|}{ Shape } \\
\hline Oval & 1 \\
\hline Round & 0 \\
\hline Irregular & 26 \\
\hline \multicolumn{2}{|l|}{ Margin } \\
\hline Circumscribed & 1 \\
\hline Not circumscribed & 26 \\
\hline \multicolumn{2}{|l|}{ Internal enhancement characteristics } \\
\hline Homogeneous & 0 \\
\hline Heterogeneous & 27 \\
\hline Rim enhancement & 0 \\
\hline Dark internal septations & 0 \\
\hline Non- mass enhancement & 4 \\
\hline Non- mass enhancement and mass & 4 \\
\hline \multicolumn{2}{|l|}{ Associated features } \\
\hline Nipple retraction & 14 \\
\hline Nipple invasion & 3 \\
\hline Skin retraction & 13 \\
\hline Skin thickening & 15 \\
\hline Skin invasion & 10 \\
\hline Axillary adenopathy & 10 \\
\hline Pectoralis muscle invasion & 5 \\
\hline Chest wall invasion & 0 \\
\hline Architectural distortion & 0 \\
\hline
\end{tabular}

$A C R$ American college radiology, BI-RADS® breast imaging-reporting and data system, MRI magnetic resonance imaging, $n$ number of patients

\section{Pathological assessment}

Pathology was used as the gold standard for comparing tumour size measurements after neoadjuvant treatment. Comprehensive pathological analysis of surgical specimens was performed by an experienced breast cancer pathologist. During the course of the study supplementary scientific incisional surgical biopsies of tumour tissue were obtained, after the MRI examinations, at baseline, after 8 weeks and after surgery (Fig. 1). These scientific biopsies allowed verification of ER status, progesterone receptor (PR) status and HER-2 status.

Pathological response was defined as complete responder (no residual invasive disease was present) or not complete responder (residual invasive disease present). Total extent of residual disease was reported, measured as the greatest one- dimensional extent in centimetres of residual invasive cancer including intervening areas of fibrosis and/or necrosis. In situ components measurements were not included.

\section{Statistical analysis}

Descriptive statistics were used to compared two measurements of tumour response (changes in LD at MRI and CE) to predict pathologic outcome. Each predictor variable was measured at three time points. Missing data elements were not included in the analysis. The Spearman rank correlation was applied to study the statistical dependence between the classification of the tumour sizes estimated by CE and MRI, and pathological results. The Bland-Altman analysis was used to evaluate deviations from the mean of the measures by two methods (CE versus pathology, and MRI versus pathology) and to estimate a range of agreement, within which $95 \%$ of the differences between the measurement by CE or MRI and the pathological measurement. Box plots were conducted to visually summarized the distribution of the numerical data. 
Fig. 3 a, b Two different patients' magnetic resonance imaging examinations demonstrating response to treatment and residual tumour sizes.

Manual assessment of tumour longest diameter using the tumour tracking system incorporated in Philips IntelliSpace Portal program. Description of the time points from left to right: at baseline, between regimens (after 2 months of treatment) and presurgery (after 4 months of treatment) (a)

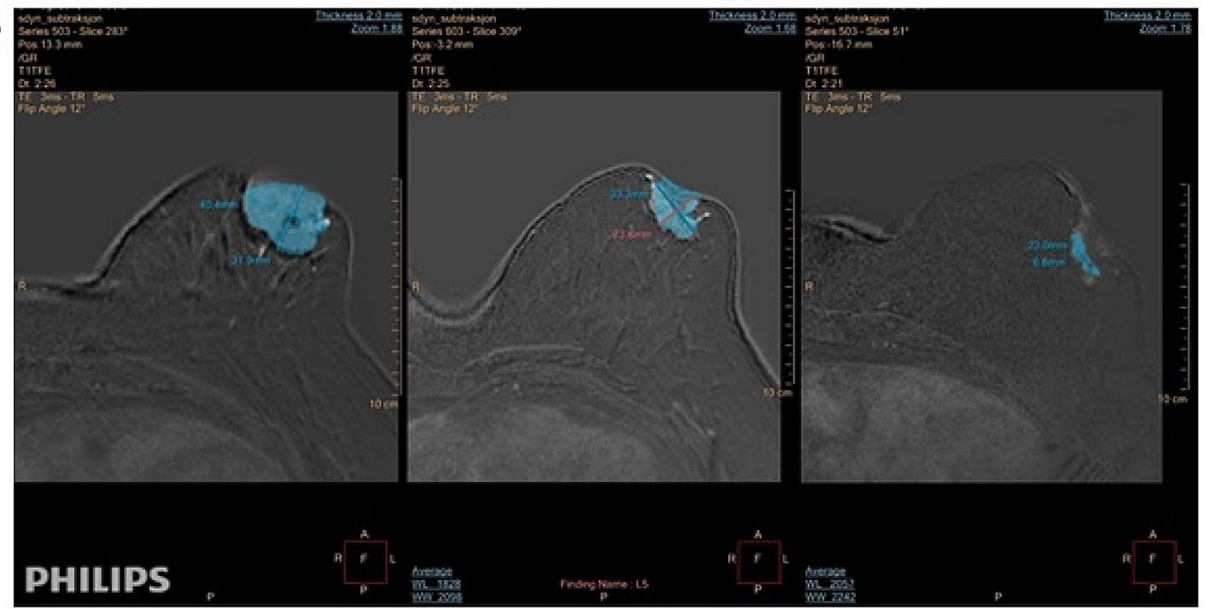

(b)

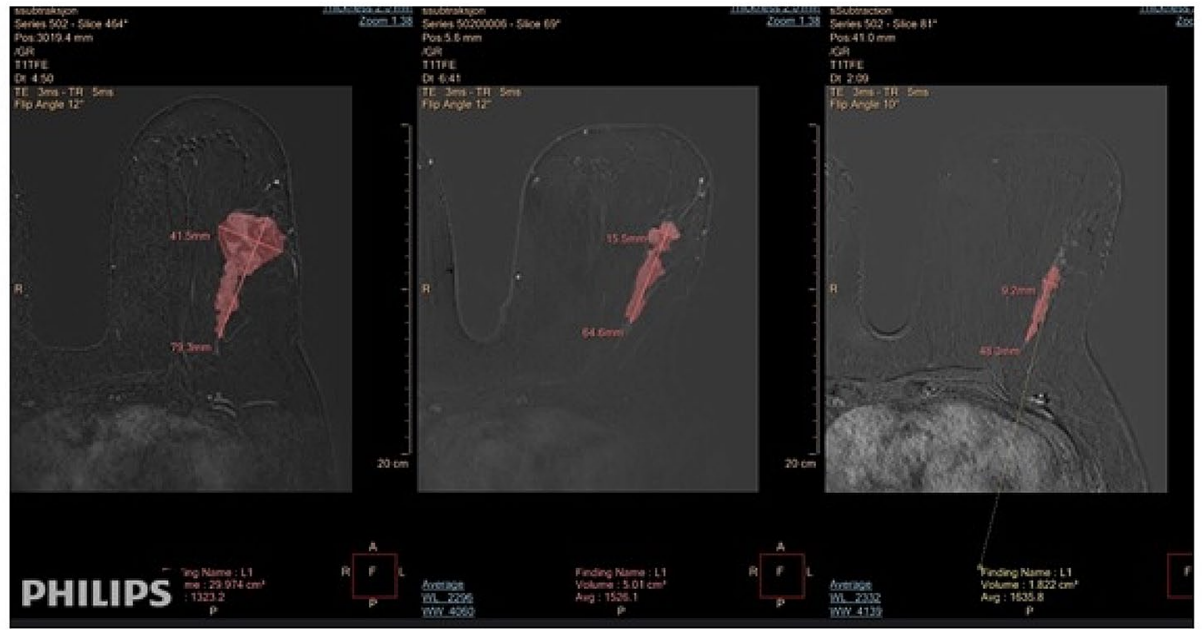

Statistical analysis was performed using $\mathrm{R}$ software. A twosided $p \leq 0.05$ was considered statistically significant.

\section{Ethical requirements}

The Regional Committee for Medical and Health Research Ethics South-East Norway approved the study protocol. Informed consent was obtained from all individual participants included in the study. The imaging findings have not previously been reported.

\section{Results}

The clinical-based TNM classification was used to identify 29 cases of T4 tumours (82.9\%) and 6 cases of T3 tumours (17.1\%) (Table 1).

\section{Baseline comparison of tumour size before starting therapy}

The median tumour size (LD) at baseline on MRI was $3.4 \mathrm{~cm}$ (interquartile range $2.9-5.0 \mathrm{~cm}$ ); on $\mathrm{CE}$ was
$5.0 \mathrm{~cm}$ (interquartile range $4.0-6.0 \mathrm{~cm}$ ). Spearman correlation showed that baseline tumour size on CE and MRI had a strong positive agreement $(r=0.71)$, (Fig. 4a). The Bland-Altman plot was used to measure the difference of pre-surgical tumour sizes against the mean value of MRI and clinical assessments. The Bland-Altman (Fig. 4b) analysis indicated that the average lesion size estimated by MRI was smaller than compared with CE. The mean difference between the two measurements was $1.06 \mathrm{~cm}$; with a 95\% confidence interval (CI) of $0.63-1.49 \mathrm{~cm}(p \leq 0)$.

\section{Between- regimens time point comparison of tumour size}

The median tumour size between regimens on MRI was $2.7 \mathrm{~cm}$ (interquartile range $1.7-3.6 \mathrm{~cm}$ ); on $\mathrm{CE}$ was $3.0 \mathrm{~cm}$ (interquartile range $2.5-3.8 \mathrm{~cm}$ ). Spearman correlation showed that at this time point tumour size on CE and MRI had a moderate positive agreement $(r=0.53)$, (Fig. $4 \mathrm{c})$. The Bland-Altman (Fig. 4d) analysis suggested that average size estimated by MRI was greater in larger tumours and lesser 
(a)

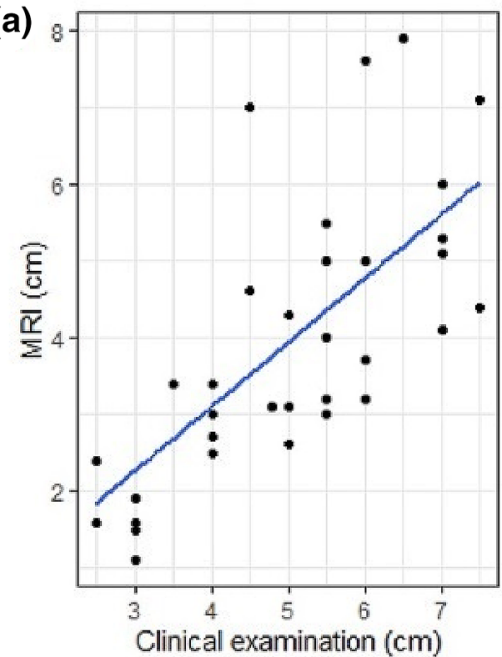

(c)

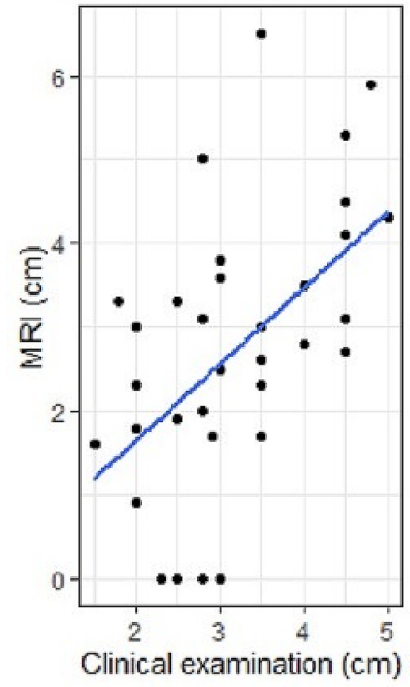

(e)

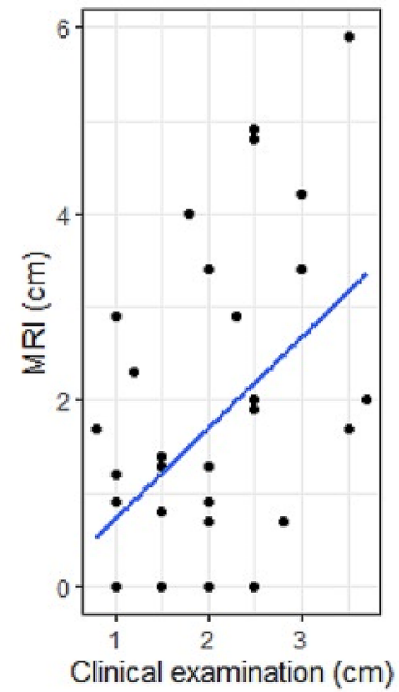

(b)

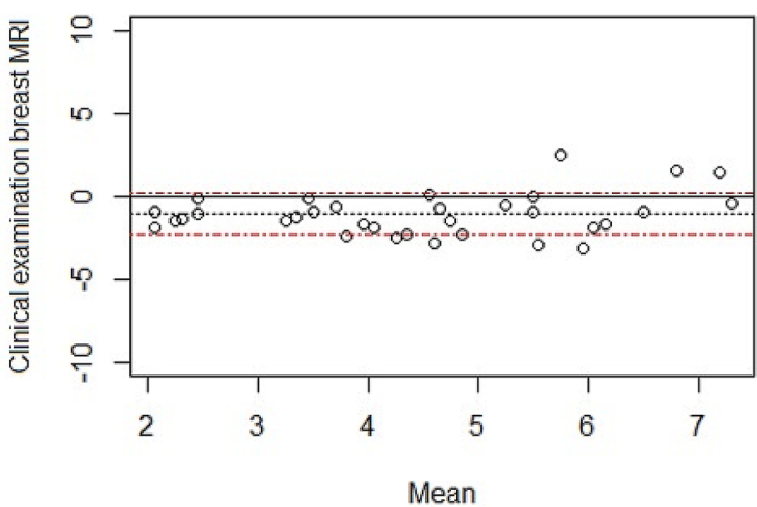

(d)

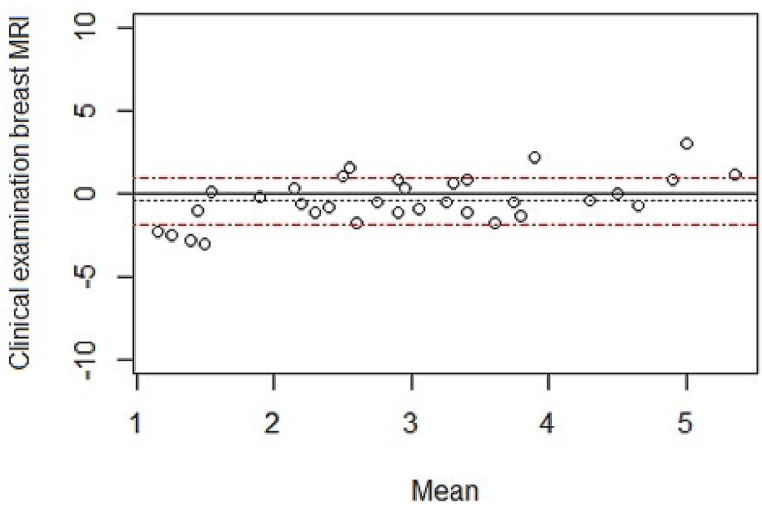

(f)

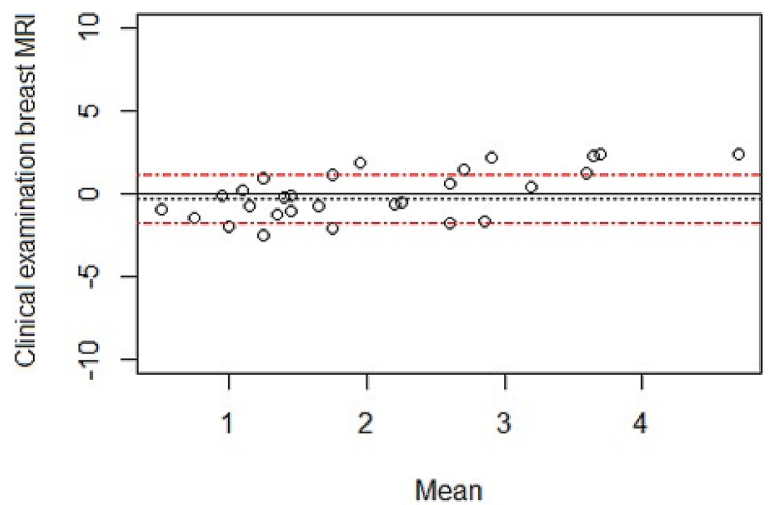


4Fig. 4 Scatterplots showing the longest diameter of tumour measured on MRI and CE, and Bland-Altman plots illustrating the size difference between the measurements by MRI and CE $(\mathbf{a}-\mathbf{f})$. a Scatterplot at baseline, b Bland-Altman plot at baseline, c Scatterplot between regimens, d Bland-Altman plot between regimens, e Scatterplot at the end of the neoadjuvant therapy and $\mathbf{f}$ Bland-Altman plot at the end of the neoadjuvant therapy. Correlation between measurements at baseline was strong $r=0.71$, between measurements was moderate $r=0.53$ and at the end of the neoadjuvant therapy was moderate $\mathrm{r}=0.46$. Trend line (black line) depicts least-squares fit for data. MRI: magnetic resonance imaging

in smaller tumours compared with $\mathrm{CE}$ (mean difference $0.45 \mathrm{~cm}, \mathrm{CI}$ of -0.04 to $0.94 \mathrm{~cm}, p=0.07)$.

\section{Comparison of tumour size after completion of therapy}

The median size of the tumours after completion of NAAI therapy on MRI was $1.3 \mathrm{~cm}$ (interquartile range $0.4-2.6 \mathrm{~cm}$ ) and on CE $2.0 \mathrm{~cm}$ (interquartile range $1.5-2.5 \mathrm{~cm}$ ), with a moderate agreement, $r=0.46$, (Fig. 4e). The Bland-Altman (Fig. 4f) analysis suggested that the average lesion size estimated by MRI was larger than compared with CE (mean difference $0.30 \mathrm{~cm}$, with a $95 \%$ CI of -0.20 to $0.80 \mathrm{~cm}$, $p=0.23$ ).

\section{Assessments outcomes and response evaluation}

Pathological tumour size median was $2.5 \mathrm{~cm}$ (interquartile range $2.0-3.0 \mathrm{~cm}$ ). The correlation between post-treatment MRI size and pathology was moderate and higher $(r=0.64$, $p=0.001)$ compared to the correlation between CE and pathology ( $r=0.25, p=0.04)$, (Fig. 5). Tumour size on MRI had $0.82 \mathrm{~cm}$ lower mean size $(95 \%$ CI of $0.37-1.27 \mathrm{~cm})$ than tumour size measured by pathology. For CE, the measured tumour size was on average $0.52 \mathrm{~cm}$ smaller (95\% CI of $0.03-1.02 \mathrm{~cm})$.

Figure 6 shows boxplots of all three measurement variables. MRI underestimated tumour size in 24 patients $(68.6 \%)$ and overestimated in 9 patients $(25.7 \%)$ compared to pathology. At completion of neoadjuvant therapy, 35 (100\%) patients in the analysis showed a clinical partial response, and in turn MRI demonstrated a partial response in 25 (71.4\%) patients, complete response in 9 (25.7\%) patients and stable disease in 1 (2.9\%). Thirty-two (91.4\%) patients in the pathological analysis were not complete responders and just $3(8.6 \%)$ patients were reported as complete pathological responders. Two of the three complete responses that were determined on pathology showed complete response at MRI. In addition, all of the three pathological complete responders were found to have clinical residual disease.

\section{Discussion}

It is of vital importance to develop effective assessments of treatment response to maximize patient benefit during neoadjuvant breast cancer therapy. The results of this study indicate that breast MRI is an accurate method for assessing early response evaluation, residual disease and surgical planning in the neoadjuvant setting. In comparison with pathology and CE, the diagnostic accuracy of MRI is stronger in assessing disease extent, screening for other foci either in the affected or in the contralateral breast, and investigating satellite tumours as a repeatable 3D assessment [37, 38]. Regardless significant improvement in MRI technique, interpretation and analysis, tumour size can be over- or underestimated [17, 39-41]. Although, unlike pathological response, early changes in tumour measures by MRI can be assessed at a stage when treatment regimen can still be modified. Pathological response is strictly an endpoint and cannot be used to improve treatment planning [16, 42].

Imaging measurements are aggregations of all tumour tissue that meet criteria for signal enhancement and are dependent on parameters of the image's acquisitions. These parameters include timing relative to contrast agent injection and physiologic conditions affecting the distribution of the contrast agent.

Furthermore, the effects in tumour vascularity induced by anti- angiogenetic effects in response to neoadjuvant therapy may explain the decrease or delay in imaging enhancement, demonstrating greater bias in residual tumour measurement. Reactive inflammation, fibrosis or necrosis are difficult to distinguish from residual tumour, and measurement errors may be cumulative when tumours regress as multiple, scattered deposits [19, 43].

In our sample population MRI tended to underestimate the actual tumour size in $68.6 \%$ of the cases and overestimated the pathology size in $25.7 \%$ of the cases directly prior to surgery.

Seven cases were discordantly diagnosed as imaging complete response but non-pathological complete response after NAAI therapy. The decrease in enhancement secondary to the changes in tumour vascularization induced by the anti-angiogenetic effect of therapeutic agents also contributed to the underestimation of MRI in the prediction of residual tumour size, and a high rate of false- negative interpretations [44]. After therapy, an enhanced area was no longer detected by MRI thus, the tumour response was considered complete. However, pathological analysis of the surgical specimen revealed microscopically scattered residual cancer nests in the tumour bed, indicating a nonpathological complete response.

The overestimation percentage is consistent with the literature, ranging from 6 to $81 \%$ [45-47]. We found that 


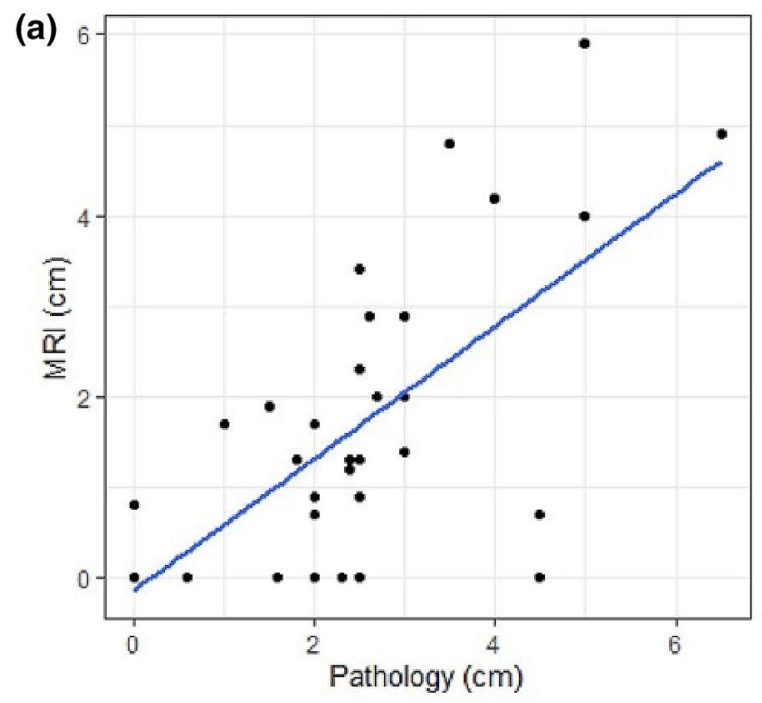

Fig. 5 a, b Scatterplots showing longest diameter of tumour measured on MRI and pathology $\mathbf{a}$ and by CE and pathology $\mathbf{b}$ at the the end of the neoadjuvant therapy and after surgery. Correlation between

MRI diagnosed only one case of pathological complete response as a non-imaging complete response. Fibrous granulation tissue that may have caused the misdiagnosis that was observed in that case. MRI after NAAI therapy showed a small mass measuring less than $1 \mathrm{~cm}$ in diameter with scattered enhancement, interpreted as an imaging non-complete response. Pathology, however, showed fibrous granulation tissue in the surgical specimen without residual cancer cells, indicating pathological complete response. The fibrous granulation tissue contained inflammatory cells and numerous small vessels.

Mennella et al. found that the main reason for discordance between MRI and pathological measures is ductal carcinoma in situ (DCIS) [48]. MRI measurement included both invasive and non-invasive components, while it is well accepted that pathologists consider just the invasive component size of the tumour. Thus, DCIS found in MRI may be one of the reasons for overestimation of the size of the invasive tumour in some cases presented in this study [49].

Weatherall et al. found that the sampling error between pathological and MRI measurements decreased when the lesion diameter was obtained by averaging measurements made in three orthogonal dimensions [50]. This approach was not used in our study, but it may help to further improve the reproducibility and accuracy of our measurements.

$\mathrm{CE}$ was found to have a less dispersed data, and like MRI, it frequently underestimated size in comparison with pathology in our population. Clinical assessment was found to be less accurate for assessing complete responses, with a lower correlation with pathological measurements and 3 false-positive interpretations. Although, $\mathrm{CE}$ is essential, it is
MRI and pathology was moderate and stronger $r=0.64$, then between $\mathrm{CE}$ and pathology, $r=0.25$. MRI Magnetic resonance imaging

inherently subjective and cannot be validated as a definitive and exclusive assessment method.

This current study should be considered in the context of its strengths and limitations. The first limitation of our study was the small sample size. However, this cohort of patients represents a pilot sub-study of an ongoing trial. A second limitation was that the imaging-based measurements were finally reviewed in a consensus imaging interpretation, and thus, interobserver variability was not evaluated. The major strength is that there are no previous studies analysing MRI accuracy measuring treatment response in patients receiving NAAI therapy. Another strength is the consecutive prospective inclusion of patients. With an increasing use of NAAI therapy it is of particular interest to determine the most accurate of the current assessment methods to evaluate at the earliest the treatment effect.

In conclusion, our results showed that in postmenopausal patients diagnosed with $\mathrm{LABC}$ treated neoadjuvant with NAAI, the correlation between post-treatment MRI size and pathology was higher compared to the correlation between $\mathrm{CE}$ and pathology. MRI was found to be more accurate for estimating complete responses then clinical assessments. The results of this study provide further support for the benefit of a clinico-imaging preoperative assessment for evaluation of response, residual disease and the importance in deciding patient's eligibility for BCS. Guidelines to correlate imaging and clinicopathological assessments, and further studies with larger sample sizes are needed.

Acknowledgements The authors acknowledge the NEOLETEXE Trial Team. All the patients who participated in the study. The administration 


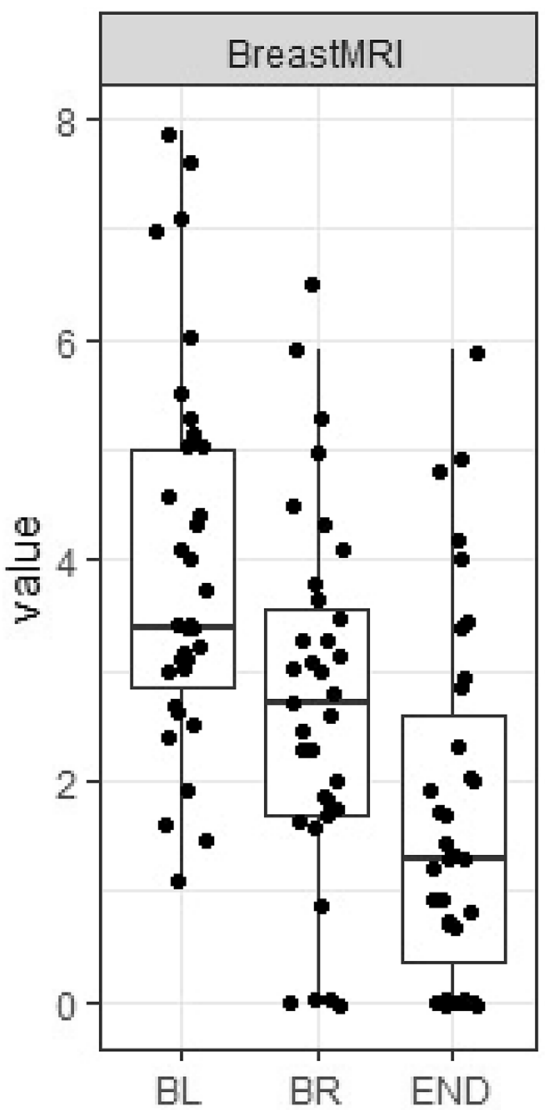

BL
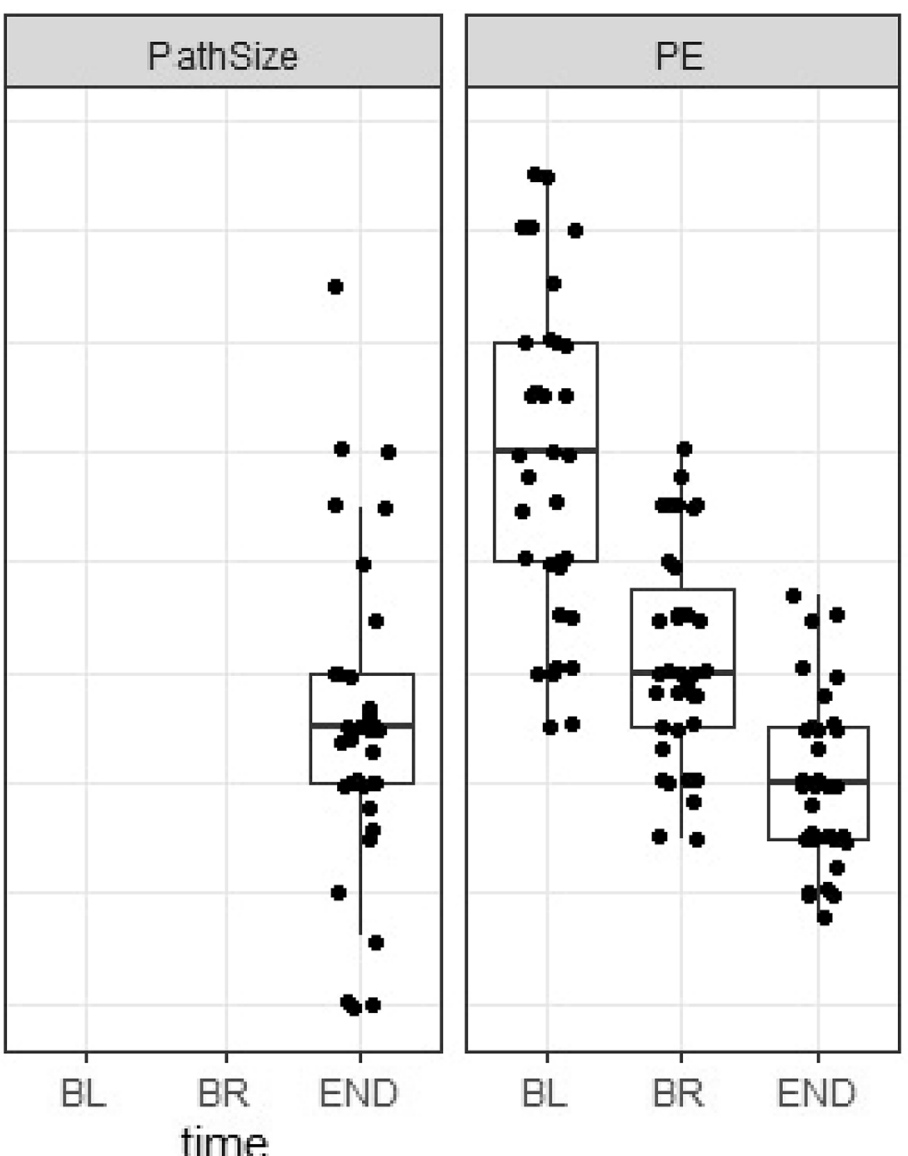

Fig. 6 Comparison of the residual tumour size at baseline, between regimens and at the end of neoadjuvant therapy on MRI and CE. In addition, comparison of residual tumour size at the end of neoadjuvant therapy between MRI, pathological and clinical measurements. Box plots illustrating the median size of the three variables, with the

of the Department of Diagnostic Imaging and Intervention at Akershus University Hospital for a scientific grant allowing this work to be conducted as part of the $\mathrm{PhD}$ thesis of J. Reis. The authors also gratefully acknowledge Aida Lunder, $\mathrm{PhD}$, for her critical advice and constantly assistance to this study.

Funding The authors state that this work has not received any funding. Open access funding provided by Akershus University Hospital (AHUS)

\section{Compliance with ethical standards}

Conflict of interest The authors of this manuscript declare no relationships with any companies, whose products or services may be related to the subject matter of the article.

Statistics and biometry One of the authors has significant statistical expertise. No writing assistance was utilized in the production of this manuscript. corresponding interquartile range and whiskers from the 5 th to the 95th percentile. $B L$ Baseline, $B R$ between regimens, $E N D$ the end of therapy, MRI magnetic resonance imaging, PathSize pathological size, $P E$ physical/CE

Ethical approval Institutional Review Board approval was obtained. All procedures performed in studies involving human participants were in accordance with the ethical standards of the institutional and/ or national research committee and with the 1964 Helsinki declaration and its later amendments or comparable ethical standards.

Informed consent Written informed consent was obtained from all subjects (patients) in this study.

Open Access This article is licensed under a Creative Commons Attribution 4.0 International License, which permits use, sharing, adaptation, distribution and reproduction in any medium or format, as long as you give appropriate credit to the original author(s) and the source, provide a link to the Creative Commons licence, and indicate if changes were made. The images or other third party material in this article are included in the article's Creative Commons licence, unless indicated otherwise in a credit line to the material. If material is not included in the article's Creative Commons licence and your intended use is not permitted by statutory regulation or exceeds the permitted use, you will need to obtain permission directly from the copyright holder. To view a copy of this licence, visit http://creativecommons.org/licenses/by/4.0/. 


\section{References}

1. Sannachi L, Gangeh M, Tadayyon H, Sadeghi-Naini A, Gandhi S, Wright FC, Slodkowska E, Curpen B, Tran W, Czarnota GJ (2018) Response monitoring of breast cancer patients receiving neoadjuvant chemotherapy using quantitative ultrasound, texture, and molecular features. PLoS ONE 13(1):e0189634

2. Fowler AM, Mankoff DA, Joe BN (2017) Imaging neoadjuvant therapy response in breast cancer. Radiology 285(2):358-375

3. Provencher L, Hogue JC, Desbiens C, Poirier B, Poirier E, Boudreau D, Joyal M, Diorio C, Duchesne N, Chiquette J (2016) Is clinical breast examination important for breast cancer detection? Curr Oncol 23(4):e332-339

4. Bodini M, Berruti A, Bottini A, Allevi G, Fiorentino C, Brizzi MP, Bersiga A, Generali D, Volpi D, Marini U et al (2004) Magnetic resonance imaging in comparison to clinical palpation in assessing the response of breast cancer to epirubicin primary chemotherapy. Breast Cancer Res Treat 85(3):211-218

5. Cortadellas T, Argacha P, Acosta J, Rabasa J, Peiro R, Gomez M, Rodellar L, Gomez S, Navarro-Golobart A, Sanchez-Mendez S et al (2017) Estimation of tumor size in breast cancer comparing clinical examination, mammography, ultrasound and MRI-correlation with the pathological analysis of the surgical specimen. Gland Surg 6(4):330-335

6. Cordoba O, Carrillo-Guivernau L, Reyero-Fernandez C (2018) Surgical management of breast cancer treated with neoadjuvant therapy. Breast Care (Basel) 13(4):238-243

7. Scheel JR, Kim E, Partridge SC, Lehman CD, Rosen MA, Bernreuter WK, Pisano ED, Marques HS, Morris EA, Weatherall PT et al (2018) MRI, Clinical examination, and mammography for preoperative assessment of residual disease and pathologic complete response after neoadjuvant chemotherapy for breast cancer: ACRIN 6657 Trial. AJR Am J Roentgenol 210(6):1376-1385

8. Prati R, Minami CA, Gornbein JA, Debruhl N, Chung D, Chang HR (2009) Accuracy of clinical evaluation of locally advanced breast cancer in patients receiving neoadjuvant chemotherapy. Cancer 115(6):1194-1202

9. Hylton NM, Blume JD, Bernreuter WK, Pisano ED, Rosen MA, Morris EA, Weatherall PT, Lehman CD, Newstead GM, Polin S et al (2012) Locally advanced breast cancer: MR imaging for prediction of response to neoadjuvant chemotherapy—results from ACRIN 6657/I-SPY TRIAL. Radiology 263(3):663-672

10. Menezes GL, Knuttel FM, Stehouwer BL, Pijnappel RM, van den Bosch MA (2014) Magnetic resonance imaging in breast cancer: a literature review and future perspectives. World J Clin Oncol 5(2):61-70

11. Esserman L, Hylton N, Yassa L, Barclay J, Frankel S, Sickles E (1999) Utility of magnetic resonance imaging in the management of breast cancer: evidence for improved preoperative staging. J Clin Oncol 17(1):110-119

12. Van Goethem M, Schelfout K, Dijckmans L, Van Der Auwera JC, Weyler J, Verslegers I, Biltjes I, De Schepper A (2004) MR mammography in the pre-operative staging of breast cancer in patients with dense breast tissue: comparison with mammography and ultrasound. Eur Radiol 14(5):809-816

13. Berg WA, Gutierrez L, NessAiver MS, Carter WB, Bhargavan M, Lewis RS, Ioffe OB (2004) Diagnostic accuracy of mammography, clinical examination, US, and MR imaging in preoperative assessment of breast cancer. Radiology 233(3):830-849
14. Hollingsworth AB, Stough RG, O'Dell CA, Brekke CE (2008) Breast magnetic resonance imaging for preoperative locoregional staging. Am J Surg 196(3):389-397

15. Klein J, Tran W, Watkins E, Vesprini D, Wright FC, Look Hong NJ, Ghandi S, Kiss A, Czarnota GJ (2019) Locally advanced breast cancer treated with neoadjuvant chemotherapy and adjuvant radiotherapy: a retrospective cohort analysis. BMC Cancer 19(1):306

16. Kuerer HM, Newman LA, Smith TL, Ames FC, Hunt KK, Dhingra K, Theriault RL, Singh G, Binkley SM, Sneige N et al (1999) Clinical course of breast cancer patients with complete pathologic primary tumor and axillary lymph node response to doxorubicinbased neoadjuvant chemotherapy. J Clin Oncol 17(2):460-469

17. Fukuda T, Horii R, Gomi N, Miyagi Y, Takahashi S, Ito Y, Akiyama F, Ohno S, Iwase T (2016) Accuracy of magnetic resonance imaging for predicting pathological complete response of breast cancer after neoadjuvant chemotherapy: association with breast cancer subtype. Springerplus 5:152

18. Marinovich ML, Houssami N, Macaskill P, Sardanelli F, Irwig L, Mamounas EP, von Minckwitz G, Brennan ME, Ciatto S (2013) Meta-analysis of magnetic resonance imaging in detecting residual breast cancer after neoadjuvant therapy. J Natl Cancer Inst 105(5):321-333

19. Eisenhauer EA, Therasse P, Bogaerts J, Schwartz LH, Sargent D, Ford R, Dancey J, Arbuck S, Gwyther S, Mooney $M$ et al (2009) New response evaluation criteria in solid tumours: revised RECIST guideline (version 1.1). Eur J Cancer 45(2):228-247

20. Irwig L, Bennetts A (1997) Quality of life after breast conservation or mastectomy: a systematic review. Aust N Z J Surg 67(11):750-754

21. Boughdad S, Champion L, Becette V, Cherel P, Fourme E, Lemonnier J, Lerebours F, Alberini JL (2020) Early metabolic response of breast cancer to neoadjuvant endocrine therapy: comparison to morphological and pathological response. Cancer Imaging 20(1): 11

22. Hilal T, Covington M, Kosiorek HE, Zwart C, Ocal IT, Pockaj BA, Northfelt DW, Patel BK (2018) Breast MRI phenotype and background parenchymal enhancement may predict tumor response to neoadjuvant endocrine therapy. Breast J 24(6):1010-1014

23. Miller WR (2003) Aromatase inhibitors: mechanism of action and role in the treatment of breast cancer. Semin Oncol 30(4 Suppl 14):3-11

24. Cardoso F, Kyriakides S, Ohno S, Penault-Llorca F, Poortmans P, Rubio IT, Zackrisson S, Senkus E (2019) Early breast cancer: ESMO clinical practice guidelines for diagnosis, treatment and follow-up. Ann Oncol 30(8):1194-1220

25. Geisler J, Lonning PE (2005) Aromatase inhibition: translation into a successful therapeutic approach. Clin Cancer Res 11(8):2809-2821

26. Barroso-Sousa R, Silva DD, Alessi JV, Mano MS (2016) Neoadjuvant endocrine therapy in breast cancer: current role and future perspectives. Ecancermedicalscience 10:609

27. Garg PK, Prakash G (2015) Current definition of locally advanced breast cancer. Curr Oncol 22(5):e409-410

28. Shao N, Xie C, Shi Y, Ye R, Long J, Shi H, Shan Z, Thompson AM, Lin Y (2019) Comparison of the 7th and 8th edition of American Joint Committee on Cancer (AJCC) staging systems for breast cancer patients: a surveillance, epidemiology and end results (SEER) Analysis. Cancer Manag Res 11:1433-1442 
29. Dixon JM, Anderson TJ, Miller WR (2002) Neoadjuvant endocrine therapy of breast cancer: a surgical perspective. Eur J Cancer 38(17):2214-2221

30. Cardoso F, Senkus E, Costa A, Papadopoulos E, Aapro M, Andre F, Harbeck N, Aguilar Lopez B, Barrios CH, Bergh J et al (2018) 4th ESO-ESMO International Consensus Guidelines for Advanced Breast Cancer (ABC 4)dagger. Ann Oncol 29(8):1634-1657

31. Gennatas C, Michalaki V, Carvounis E, Psychogios J, Poulakaki N, Katsiamis G, Voros D, Kouloulias V, Mouratidou D, Tsavaris $\mathrm{N}$ (2006) Third-line hormonal treatment with exemestane in postmenopausal patients with advanced breast cancer progressing on letrozole or anastrozole. A phase II trial conducted by the Hellenic Group of Oncology (HELGO). Tumori 92(1):13-17

32. Spring LM, Gupta A, Reynolds KL, Gadd MA, Ellisen LW, Isakoff SJ, Moy B, Bardia A (2016) Neoadjuvant endocrine therapy for estrogen receptor-positive breast cancer: a systematic review and meta-analysis. JAMA Oncol 2(11):1477-1486

33. Eiermann W, Paepke S, Appfelstaedt J, Llombart-Cussac A, Eremin J, Vinholes J, Mauriac L, Ellis M, Lassus M, ChaudriRoss HA et al (2001) Preoperative treatment of postmenopausal breast cancer patients with letrozole: A randomized double-blind multicenter study. Ann Oncol 12(11):1527-1532

34. Leal F, Liutti VT, Antunes dos Santos VC, Novis de Figueiredo MA, Macedo LT, Rinck Junior JA, Sasse AD (2015) Neoadjuvant endocrine therapy for resectable breast cancer: a systematic review and meta-analysis. Breast 24(4):406-412

35. Smith IE, Dowsett M, Ebbs SR, Dixon JM, Skene A, Blohmer JU, Ashley SE, Francis S, Boeddinghaus I, Walsh G et al (2005) Neoadjuvant treatment of postmenopausal breast cancer with anastrozole, tamoxifen, or both in combination: the immediate preoperative anastrozole, tamoxifen, or combined with tamoxifen (IMPACT) multicenter double-blind randomized trial. J Clin Oncol 23(22):5108-5116

36. Bahrami N, Sauer T, Engebretsen S, Aljabri B, Bemanian V, Lindstrom J, Luders T, Kristensen V, Lorentzen A, Loeng M et al (2019) The NEOLETEXE trial: a neoadjuvant cross-over study exploring the lack of cross resistance between aromatase inhibitors. Future Oncol 15(32):3675-3682

37. Van Goethem M, Schelfout K, Kersschot E, Colpaert C, Verslegers I, Biltjes I, Tjalma WA, De Schepper A, Weyler J, Parizel PM (2007) MR mammography is useful in the preoperative locoregional staging of breast carcinomas with extensive intraductal component. Eur J Radiol 62(2):273-282

38. Lehman CD, Gatsonis C, Kuhl CK, Hendrick RE, Pisano ED, Hanna L, Peacock S, Smazal SF, Maki DD, Julian TB et al (2007) MRI evaluation of the contralateral breast in women with recently diagnosed breast cancer. N Engl J Med 356(13):1295-1303

39. Jiang YZ, Xia C, Peng WT, Yu KD, Zhuang ZG, Shao ZM (2014) Preoperative measurement of breast cancer overestimates tumor size compared to pathological measurement. PLoS ONE 9(1):e86676

40. Grimsby GM, Gray R, Dueck A, Carpenter S, Stucky CC, Aspey H, Giurescu ME, Pockaj B (2009) Is there concordance of invasive breast cancer pathologic tumor size with magnetic resonance imaging? Am J Surg 198(4):500-504

41. Woodhams R, Kakita S, Hata H, Iwabuchi K, Kuranami M, Gautam S, Hatabu H, Kan S, Mountford C (2010) Identification of residual breast carcinoma following neoadjuvant chemotherapy: diffusion-weighted imaging-comparison with contrast-enhanced MR imaging and pathologic findings. Radiology 254(2):357-366

42. Bonadonna G, Valagussa P, Brambilla C, Ferrari L, Moliterni A, Terenziani M, Zambetti M (1998) Primary chemotherapy in operable breast cancer: eight-year experience at the Milan Cancer Institute. J Clin Oncol 16(1):93-100

43. Yeh E, Slanetz P, Kopans DB, Rafferty E, Georgian-Smith D, Moy L, Halpern E, Moore R, Kuter I, Taghian A (2005) Prospective comparison of mammography, sonography, and MRI in patients undergoing neoadjuvant chemotherapy for palpable breast cancer. AJR Am J Roentgenol 184(3):868-877

44. Wu WP, Wu HK, Chen CJ, Lee CW, Chen ST, Chen DR, Chou CT, Mok CW, Lai HW (2019) Higher underestimation of tumour size post-neoadjuvant chemotherapy with breast magnetic resonance imaging (MRI)-A concordance comparison cohort analysis. PLoS ONE 14(10):e0222917

45. Behjatnia B, Sim J, Bassett LW, Moatamed NA, Apple SK (2010) Does size matter? Comparison study between MRI, gross, and microscopic tumor sizes in breast cancer in lumpectomy specimens. Int J Clin Exp Pathol 3(3):303-309

46. Chandwani S, George PA, Azu M, Bandera EV, Ambrosone CB, Rhoads GG, Demissie K (2014) Role of preoperative magnetic resonance imaging in the surgical management of early-stage breast cancer. Ann Surg Oncol 21(11):3473-3480

47. Leddy R, Irshad A, Metcalfe A, Mabalam P, Abid A, Ackerman S, Lewis M (2016) Comparative accuracy of preoperative tumor size assessment on mammography, sonography, and MRI: Is the accuracy affected by breast density or cancer subtype? J Clin Ultrasound 44(1): 17-25

48. Mennella S, Garlaschi A, Paparo F, Perillo M, Celenza M, Massa T, Rollandi GA, Garlaschi G (2015) Magnetic resonance imaging of breast cancer: factors affecting the accuracy of preoperative lesion sizing. Acta Radiol 56(3):260-268

49. Lehman CD (2010) Magnetic resonance imaging in the evaluation of ductal carcinoma in situ. J Natl Cancer Inst Monogr 2010(41):150-151

50. Weatherall PT, Evans GF, Metzger GJ, Saborrian MH, Leitch AM (2001) MRI vs. histologic measurement of breast cancer following chemotherapy: comparison with x-ray mammography and palpation. J Magn Reson Imaging 13(6):868-875

Publisher's Note Springer Nature remains neutral with regard to jurisdictional claims in published maps and institutional affiliations. 


\section{Affiliations}

\section{Joana Reis ${ }^{1,2} \oplus$. Jonas Christoffer Lindstrøm ${ }^{2,3}$. Joao Boavida ${ }^{1}$ Kjell-Inge Gjesdal ${ }^{1,4}$. Daehoon Park ${ }^{5}$. Nazli Bahrami ${ }^{6,7,8}$. Manouchehr Seyedzadeh ${ }^{1} \cdot$ Woldegabriel A. Melles $^{1} \cdot$ Torill Sauer $^{2,8,9}$ • Jürgen Geisler ${ }^{2,7,8}$. Jonn Terje Geitung ${ }^{1,2}$}

1 Department of Diagnostic Imaging and Intervention, Akershus University Hospital (AHUS), Postboks 1000, 1478 Lørenskog, Norway

2 Institute of Clinical Medicine, Campus AHUS, University of Oslo, Postboks 1000, 1478 Lørenskog, Norway

3 Health Services Research Unit, Akershus University Hospital (AHUS), Postboks 1000, 1478 Lørenskog, Norway

4 Sunnmøre MR-Clinic, Agrinorbygget, Langelansveg 15, 6010 Ålesund, Norway

5 Department of Pathology, Oslo University Hospital (OUS), Postboks 4956, 0424 Nydalen, Norway
6 Department of Breast and Endocrine Surgery, Akershus University Hospital (AHUS), Postboks 1000, 1478 Lørenskog, Norway

7 Department of Oncology, Akershus University Hospital (AHUS), Postboks 1000, 1478 Lørenskog, Norway

8 Translational Cancer Research Group, Akershus University Hospital (AHUS), Postboks 1000, 1478 Lørenskog, Norway

9 Department of Pathology, Akershus University Hospital (AHUS), Postboks 1000, 1478 Lørenskog, Norway 